\title{
A novel hybrid evidential belief function-based fuzzy logic model in spatial prediction of rainfall-induced shallow landslides in the Lang Son city area (Vietnam)
}

\begin{abstract}
The main objective of this study is to investigate potential application of an integrated evidential belief function (EBF)-based fuzzy logic model for spatial prediction of rainfallinduced shallow landslides in the Lang Son city area (Vietnam). First, a landslide inventory map was constructed from various sources. Then the landslide inventory map was randomly partitioned as a ratio of 70/30 for training and validation of the models, respectively. Second, six landslide conditioning factors (slope angle, slope aspect, lithology, distance to faults, soil type, land use) were prepared and fuzzy membership values for these factors classes were estimated using the EBF. Subsequently, fuzzy operators were used to generate landslide susceptibility maps. Finally, the susceptibility maps were validated and compared using the validation dataset. The results show that the lowest prediction capability is the fuzzy SUM (76.6\%). The prediction capability is almost the same for the fuzzy PRODUCT and fuzzy GAMMA models (79.6\%). Compared to the frequency-ratio based fuzzy logic models, the EBF-based fuzzy logic models showed better result in both the success rate and prediction rate. The results from this study may be useful for local planner in areas prone to landslides. The modelling approach can be applied for other areas.
\end{abstract}

Keyword: Evidential belief function (EBF)-based fuzzy logic model; Landslides; Vietnam 